\title{
Oublier Trump et le climat
}

Le $1^{\text {er }}$ juin dernier, Donald Trump a annoncé officiellement le retrait des États-Unis de l'Accord de Paris, adopté en 2015 lors de la COP 21, qui fixe les grandes orientations de la gouvernance climatique mondiale. Cette séquence diplomatique vient conclure un ensemble d'initiatives qui allait dans le sens d'une obstruction aux politiques de lutte contre les changements climatiques. Ainsi, le 28 mars 2017, Trump signait un décret qui balayait l'essentiel de la politique climatique programmée par Barack Obama, dont la mesure phare consistait en une réglementation des émissions de $\mathrm{CO}_{2}$ des centrales électriques fonctionnant au charbon. Le $1^{\mathrm{er}}$ juin, Trump a aussi promis de supprimer ou considérablement réduire les financements pour le changement climatique au bénéfice des institutions des Nations unies. Il a décidé la cessation des versements de son pays au bénéfice du Fonds vert pour le climat, créé en 2010, à la suite de la COP 15 . Les conséquences pourraient aussi être rudes pour la pérennité des travaux du Groupe d'experts intergouvernemental sur l'évolution du climat (GIEC), dont les États-Unis ont toujours été le plus important contributeur financier, assurant à eux seuls, aujourd'hui, près de $45 \%$ de ses coûts de fonctionnement. Cette mise en cause de la science du climat est, là encore, attestée par d'autres déclarations et décisions de Trump: le 6 novembre 2012, alors qu'il n'était pas encore président des États-Unis, il écrivait dans un tweet que «Le concept de réchauffement global a été inventé par et pour les Chinois de manière à rendre l'économie manufacturière américaine non compétitive». En décembre 2016, Trump nommait, à la tête de l'agence américaine de protection de l'environnement (EPA), Scott Pruitt, un climatosceptique notoire, dont les liens avec les lobbies pétroliers sont avérés. Il a aussi proposé de réduire le budget de cette agence de $30 \%$, et celui de la National Oceanic and Atmospheric Administration (NOAA), première agence américaine de recherche sur le climat, de $17 \%$.

C'est parce que l'Accord de Paris est «très inéquitable» et qu'il impose un intolérable «fardeau économique et financier» à son pays que Trump a décidé que les États-Unis cesseraient sa mise en œuvre. Cette attitude s'inscrit dans la ligne de celle adoptée par les précédents présidents américains qui ont toujours considéré que la question du changement climatique était, avant tout, un problème économique, d'emplois et de compétitivité internationale. Au moment du premier Sommet de la Terre de Rio, George Bush père déclarait ainsi que "Le niveau de vie des Américains n'est pas négociable». Adopté en 1997, le protocole de Kyoto était d'inspiration américaine - les conseillers économiques de Bush avaient souligné, dès le début des années 1990, que les systèmes de permis négociables apparaissent moins contraignants qu'une approche réglementaire ou fiscale - et permettait de retarder les prises de décision fortes. En juin 1997, les sénateurs Byrd et Hagel avaient fait voter à l'unanimité une motion affirmant que les États-Unis ne devaient signer aucun protocole sur le climat qui pourrait porter atteinte à l'économie sans engagement des pays du Sud. "Le mode de vie américain, c'est sacré», dira à son tour George W. Bush, lorsqu'il justifiera sa décision, en mars 2001, de ne pas soumettre la ratification du protocole de Kyoto au Sénat américain. Et le plaidoyer d'Obama, lors de son discours d'investiture le 20 janvier 2009, était enflammé: «Nous n'allons pas nous excuser pour notre mode de vie, nous le défendrons sans relâche ».

L'article 28 de l'Accord de Paris précise que sa dénonciation ne peut être notifiée que trois ans après son entrée en vigueur, intervenue le 4 novembre 2016 , avec effet du retrait une année plus tard. Ce n'est donc pas avant novembre 2020 que, suite à la décision de Trump, les États-Unis pourraient ne plus être comptés parmi les signataires de l'Accord de Paris. Mais, dans les faits, le président américain n'a pas besoin de dénoncer officiellement l'Accord, car celuici n'est pas contraignant, il repose sur des promesses, des engagements volontaires nationaux (les fameuses «contributions nationalement déterminées», INDCs en anglais), et laisse les États libres de faire ce qu'ils veulent. C'est bien ce changement d'approche relativement au protocole de Kyoto, qui imposait aux seuls pays du Nord des objectifs contraignants en matière de réduction des émissions de gaz à effet de serre, qui a permis qu'il soit adopté lors de la COP 21, un an après que l'ait été un accord climatique bilatéral signé par les États-Unis et la Chine, reposant sur la même philosophie. En d'autres termes, de Kyoto à Paris, on a 
observé un déplacement de la diplomatie climatique du juridique vers le politique - ce qu'a bien compris Trump.

On le savait dès son adoption, la mise en œuvre de l'Accord de Paris et le renforcement de son ambition seront une formidable bataille pour tout le $\mathrm{XXI}^{\mathrm{e}}$ siècle. Todd Stern, le négociateur américain pour la COP 21, ne disait pas autre chose quand il parlait d'un accord, nécessairement imparfait, qui ne peut se concevoir que comme le premier d'une série de deals qui devront être conduits bien après 2020. Dans cet esprit, on n'a peutêtre pas assez souligné que cet accord signifie la fin de ce que l'on a appelé un "exceptionnalisme climatique», une sorte de mur coupe-feu entre le climat et les autres questions qu'affrontent les sociétés, indiquant que le risque climatique ne ressemble à aucune autre et est plus menaçant que les autres. Les changements à entreprendre ne se limiteront pas au seul climat, ils doivent porter aussi sur les modes de production et de consommation, les modes de transport et de vie. Dès lors, si la décision de Trump va assurément occasionner des dégâts, sa mauvaise volonté ne pourra pas tout chambouler. Les chemins infinis de la décarbonisation ${ }^{1}$ ne dépendent pas seulement des princes qui nous gouvernent. Ayant des dimensions territoriales et locales, enchevêtrés aux conditions d'existence des individus, ils seront longs et tortueux, conflictuels et contradictoires, faits d'avancées et de reculs... Mais la transition écologique bas carbone est engagée. De l'avis des entrepreneurs et des investisseurs du secteur des renouvelables, comme de multiples acteurs, en particulier les villes et régions, les évolutions en cours ne peuvent être renversées. Avec ou sans Trump. Et en n'ayant pas uniquement en tête les questions climatiques.

Il faut, en effet, prendre la mesure des défis à affronter: la transition bas carbone n'est pas seulement une affaire de technologies pour préserver le climat. L'accord climatique États-Unis/Chine, signé en novembre 2014 -avec des promesses de réduction d'émissions confirmées à Paris en 2015-, conduirait à une

\footnotetext{
${ }^{1}$ Damian M., 2015. Les chemins infinis de la décarbonisation, Paris, Campus Ouvert/L'Harmattan.
}

convergence des émissions des deux plus gros pollueurs autour de 12 tonnes par tête de $\mathrm{CO}_{2}$ en 2030 (contre respectivement un peu plus de 17 et 6, aujourd'hui). Oublions le mode de vie sino-américain. Mais la généralisation des modes de vie et niveaux d'émissions actuels de pays développés faibles émetteurs, comme la Suède ou la France - avec respectivement 4,6 et 5 tonnes par habitant-, est tout aussi inenvisageable. Même une convergence des émissions par tête inférieure de moitié à celles de chaque Suédois ou Français pour les 10 milliards d'habitants de la planète en 2050 conduirait encore à un total mondial de $25 \mathrm{Gt}$ de $\mathrm{CO}_{2}$. Absolument intenable. Alors que l'objectif pour maintenir le réchauffement en dessous de $2{ }^{\circ} \mathrm{C}$ sur le siècle est de ramener les $40 \mathrm{Gt} /$ an d'émissions actuelles à zéro d'ici 2050, puis d'avoir, à l'horizon 2100 -ambition homérique ou farfelue, c'est selon-, des émissions «négatives », en diminuant par pompage la concentration de gaz à effet de serre dans l'atmosphère...

La question du mode de vie consumériste est explosive. Elle a été posée, sans guère d'écho jusqu'à présent - dans des termes moraux ou religieux que l'on peut ne pas partager, par le Premier ministre indien Narendra Modi : «L'aspect sur lequel j'attire l'attention de la communauté internationale est que nous devons changer nos modes de vie», mais aussi depuis Rome par la voix du pape François: «l'obsession d'un style de vie consumériste ne pourra que provoquer violence et destruction réciproque, surtout quand seul un petit nombre peut se le permettre ». Elle pourrait bien être un jour tout en haut de l'agenda des négociations dites - pour combien de temps encore ? - «climatiques». Les questions d'équité et de justice ${ }^{2}$, tout comme celles de la «décroissance», de la «satisfaisance» (sufficiency) et d'inégalités sociales et écologiques à peu près «soutenables», demeurent sous le boisseau, impensées. Pour combien de temps? Plus que jamais, il importe de penser autrement la question climatique ${ }^{3}$.

Franck-Dominique Vivien et Michel Damian

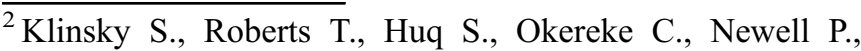
Dauvergne P., O’Brien K., Schroeder H., Tschakert P., Clapp J., Keck M., Biermann F., Liverman D., Gupta J., Rahman A., Messner D., Pellow D., Bauer S. 2017 Why equity is fundamental in climate change policy research, Global Environmental Change, 44, 170-173.

${ }^{3}$ Aubertin C., Damian M., Magny M., Millier C., Theys J., Treyer S. (Eds), 2015. Les enjeux de la Conférence de Paris. Penser autrement la question climatique, NSS, 23, $\mathrm{n}^{\circ}$ spécial, juin. Voir aussi Treyer S., Aubertin C., 2016. Les Conventions climat et biodiversité : une nouvelle géopolitique des rapports de force, NSS, 24, 4, 317-318.
} 